OPEN ACCESS

Edited by:

Fahmi Himo,

Stockholm University, Sweden

Reviewed by:

Lubomir Rulisek,

Institute of Organic Chemistry and

Biochemistry (ASCR), Czechia

Per E. M. Siegbahn,

Stockholm University, Sweden

*Correspondence:

Claudio Greco

claudio.greco@unimib.it

Specialty section:

This article was submitted to

Theoretical and Computational

Chemistry,

a section of the journa

Frontiers in Chemistry

Received: 29 October 2018 Accepted: 03 December 2018

Published: 09 January 2019

Citation:

Rovaletti A, Bruschi M, Moro G, Cosentino U and Greco C (2019) The

Challenging in silico Description of

Carbon Monoxide Oxidation as Catalyzed by Molybdenum-Copper

CO Dehydrogenase

Front. Chem. 6:630.

doi: 10.3389/fchem.2018.00630

\section{The Challenging in silico Description of Carbon Monoxide Oxidation as Catalyzed by Molybdenum-Copper CO Dehydrogenase}

\author{
Anna Rovaletti ${ }^{1}$, Maurizio Bruschi ${ }^{1}$, Giorgio Moro ${ }^{2}$, Ugo Cosentino ${ }^{1}$ and Claudio Greco ${ }^{1 *}$ \\ 'Dipartimento di Scienze dell'Ambiente e della Terra, Università Degli Studi di Milano-Bicocca, Milan, Italy, ${ }^{2}$ Dipartimento di \\ Biotecnologie e Bioscienze, Università Degli Studi di Milano-Bicocca, Milan, Italy
}

Carbon monoxide (CO) is a highly toxic gas to many living organisms. However, some microorganisms are able to use this molecule as the sole source of carbon and energy. Soil bacteria such as the aerobic Oligotropha carboxidovorans are responsible for the annual removal of about $2 \times 10^{8}$ tons of $\mathrm{CO}$ from the atmosphere. Detoxification through oxidation of $\mathrm{CO}$ to $\mathrm{CO}_{2}$ is enabled by the MoCu-dependent $\mathrm{CO}$-dehydrogenase enzyme (MoCu-CODH) which-differently from other enzyme classes with similar function-retains its catalytic activity in the presence of atmospheric $\mathrm{O}_{2}$. In the last few years, targeted advancements have been described in the field of bioengineering and biomimetics, which is functional for future technological exploitation of the catalytic properties of $\mathrm{MoCu}-\mathrm{CODH}$ and for the reproduction of its reactivity in synthetic complexes. Notably, a growing interest for the quantum chemical investigation of this enzyme has recently also emerged. This mini-review compiles the current knowledge of the MoCu-CODH catalytic cycle, with a specific focus on the outcomes of theoretical studies on this enzyme class. Rather controversial aspects from different theoretical studies will be highlighted, thus illustrating the challenges posed by this system as far as the application of density functional theory and hybrid quantum-classical methods are concerned.

Keywords: molybdenum, copper, dehydrogenase, DFT, carbon monoxide

Carbon monoxide (CO) is a fatal gas to many living organisms as well as an indirect greenhouse gas in the atmosphere (Liu et al., 2018). Global CO emissions derive both from anthropogenic and natural sources (Choi et al., 2017). One of the main sinks of atmospheric CO is constituted by the soil, in which it is consumed in large amounts by microbial oxidation (Liu et al., 2018). One example of these important soil microorganisms is represented by the aerobic bacteria Oligotropha carboxidovorans. The latter is able to grow using $\mathrm{CO}$ as its sole source of carbon and energy (Hille et al., 2015). This metabolism is ascribed to the air-stable $\mathrm{Mo} / \mathrm{Cu}$-dependent $\mathrm{CO}$ dehydrogenase $\left(\mathrm{MoCu}-\mathrm{CODH}\right.$ ) enzyme that catalyzes the oxidation of $\mathrm{CO}$ to $\mathrm{CO}_{2}$ (Zhang et al., 2010).

This enzyme contains a unique active site composed by a molybdenum/copper bimetallic center (see Figure 1A). The molybdenum ion is found in a square-pyramidal geometry with one apical oxo ligand, a dithiolene ligand from the molybdopterin cytosine dinucleotide (MCD) cofactor, an equatorial oxo ligand and a sulfido ligand. The latter bridges to the copper center, which links the active site to the protein matrix by coordinating the sulfur atom of Cys388. Moreover, $\mathrm{Cu}$ is also 
coordinated to a weakly bound water molecule (Gnida et al., 2003; Rokhsana et al., 2016), and can coordinate not only CO (i.e., the physiologic substrate) but also $\mathrm{H}_{2}$. In fact, the $\mathrm{MoCu}$ $\mathrm{CODH}$ enzyme has the ability to catalyze dihydrogen oxidation, even though such hydrogenase activity is rather low (Santiago and Meyer, 1996; Wilcoxen and Hille, 2013).

The protonation state of the active site has been object of debate. In fact, experimental X-ray diffraction (XRD) results were interpreted as indicative of a $\mathrm{Mo}(=\mathrm{O}) \mathrm{OH}$ state both for the oxidized and the reduced forms of the enzyme (Dobbek et al., 2002). Differently, extended X-ray absorption fine structure (EXAFS) spectroscopy suggested the presence of a $\mathrm{Mo}^{V I}(=\mathrm{O}) \mathrm{O}$ unit and a $\mathrm{Mo}^{I V}(=\mathrm{O}) \mathrm{OH}_{2}$ unit for the oxidized and reduced states, respectively (Gnida et al., 2003). Recent data in support of the EXAFS-based hypothesis came from electron paramagnetic resonance (EPR) analysis of the $\mathrm{Mo}^{V}$ analog and from theoretical

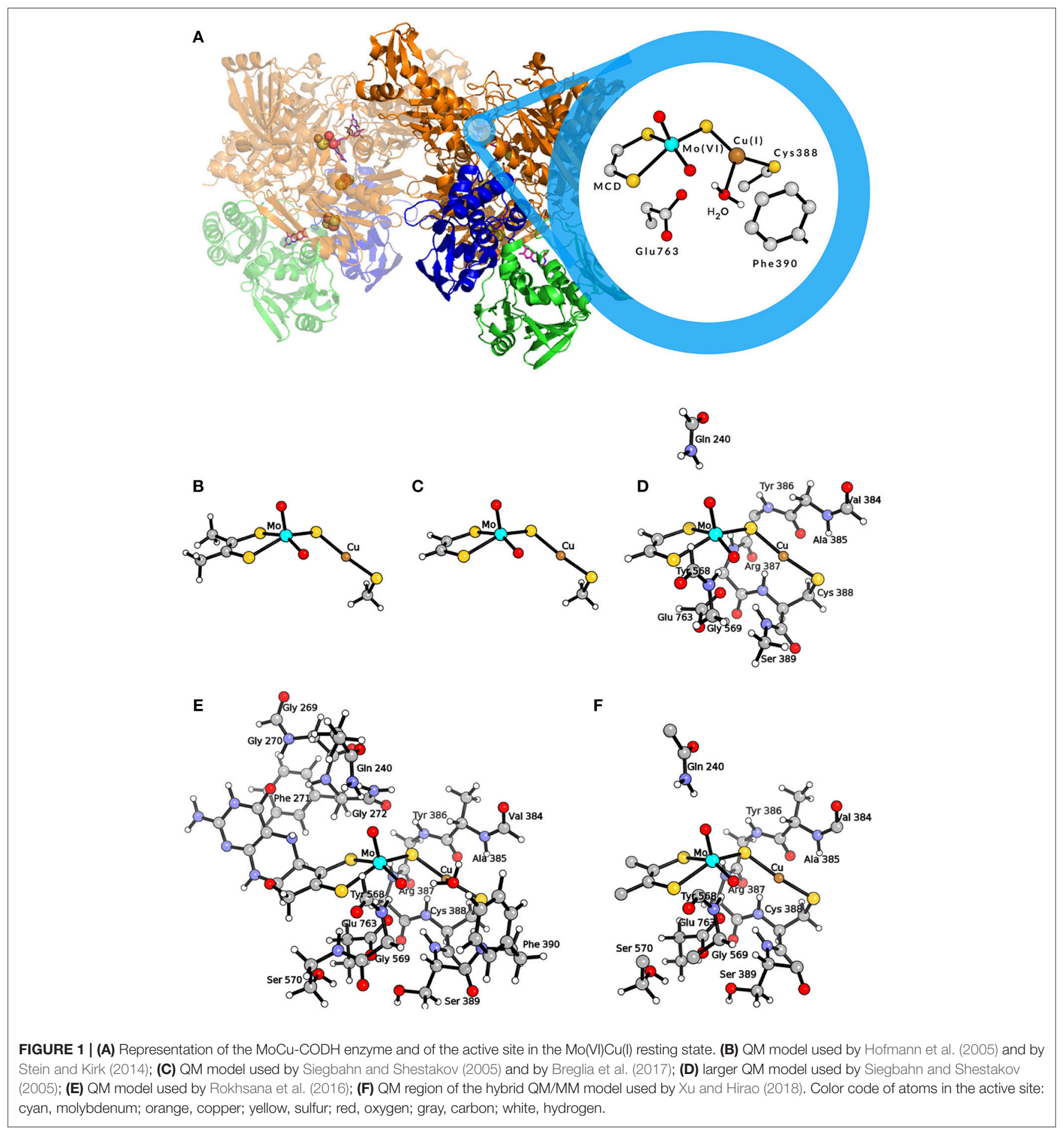


calculations (Zhang et al., 2010; Rokhsana et al., 2016). As far as the oxidation state of the $\mathrm{Cu}$ ion is concerned, it maintains the +1 state throughout the enzymatic catalytic cycle (Dobbek et al., 2002; Gnida et al., 2003). In fact, CO oxidation occurs directly at the $\mathrm{Cu}$ center (Shanmugam et al., 2013), and the twoelectron transfer to Mo at each catalytic cycle is allowed by the highly delocalized nature of the $\operatorname{Mo}(\mu-S) C u$ unit (Gourlay et al., 2006). Key second-sphere amino acid residues are conserved in $\mathrm{MoCu}-\mathrm{CODH}$ enzymes and in homologues with different activity, i.e., xanthine oxidases (Hille, 2013). In particular, a conserved glutamate residue (Glu763) is found in proximity to the equatorial oxo ligand of Mo and is considered to act as a base to facilitate deprotonation events (Wilcoxen and Hille, 2013; Hille et al., 2015). Moreover, the aromatic ring of a phenylalanine residue (Phe390), located in front of the $\mathrm{Cu}^{I}$ ion, is thought to have relevance for the correct positioning of the substrate within the active site (Rokhsana et al., 2016).

Carbon monoxide $(\mid \mathrm{C} \equiv \mathrm{O})$ is expected to show similar reactivity with respect to the isoelectronic isocyanide $(\mid \mathrm{C} \equiv \mathrm{N}$ $\mathrm{R})$ species. They share the presence of non-bonding electron pairs in the $s p$ orbital of the terminal carbon atom and a triple bond between the latter and a more electronegative atom. Indeed, Dobbek et al. reported the inhibitory activity of $n$-butylisocyanide, $\mid \mathrm{C} \equiv \mathrm{N}-\left(\mathrm{CH}_{2}\right)_{3} \mathrm{CH}_{3}$, toward the oxidized $\mathrm{MoCu}-\mathrm{CODH}$ (Dobbek et al., 2002). In the same study, the corresponding crystallographic structure of the inhibited enzyme was determined (PDB ID: 1N62). The resulting inactive complex is characterized by a thiocarbamate geometry in which the isocyanide group forms covalent bonds with the $\mu$-sulfido ligand, the equatorial oxygen of Mo and the $\mathrm{Cu}$ atom, while the alkyl chain of $n$-butylisocyanide extends into the hydrophobic interior of the substrate channel.

The features of the crystal structure of the $n$-butylisocyanidebound state prompted Dobbek and coworkers to advance the first hypothesis ever proposed for the $\mathrm{MoCu}-\mathrm{CODH}$ catalytic mechanism (see Figure 2A) (Dobbek et al., 2002). The latter involves the formation of a thiocarbonate-like intermediate-analogous to the thiocarbamate derivative formed during the aforementioned inhibition-after the CO substrate accesses the oxidized active site. Such thiocarbonate species would be characterized by the insertion of $\mathrm{CO}$ between copper, the $\mu$-sulfido ligand and the equatorial oxo ligand of the Mo atom. Taking inspiration from such a mechanistic hypothesis for CO-oxidation catalysis, the enzymatic mechanism has been subsequently studied with computational methods by several groups, thus giving birth to a debate that is still ongoing (vide infra).

Recently, it has been reported that rather bulky thiol molecules-e.g., L-cysteine, coenzyme A or glutathione-can reach the bimetallic active site (Kreß et al., 2014). They cause a reversible inactivation of the enzymatic activity, competing with the substrates for the same position at the $\mathrm{Cu}^{I}$ center.

In the following two sections of this manuscript we aim at reviewing the theoretical studies that have been published on MoCu-CODH using quantum mechanical (QM) and hybrid quantum mechanical/molecular mechanical (QM/MM) approaches. In doing so, we will pay special attention to the controversies and the challenges that have emerged. Moreover, promising future developments in the theoretical description of this system will be proposed in the concluding section.

\section{SMALL- AND MEDIUM-SIZED QM-CLUSTER MODELS}

The first theoretical investigations of the catalytic activity of $\mathrm{MoCu}-\mathrm{CODH}$ were carried out independently by two theoretical groups in 2005 (Hofmann et al., 2005; Siegbahn and Shestakov, 2005). To model the enzymatic active site, Hofmann et al. used a small cluster of 24 atoms representing the two transition metals and their first coordination spheres (see Figure 1B) (Hofmann et al., 2005), whereas Siegbahn and Shestakov performed calculations with two different models, a small one

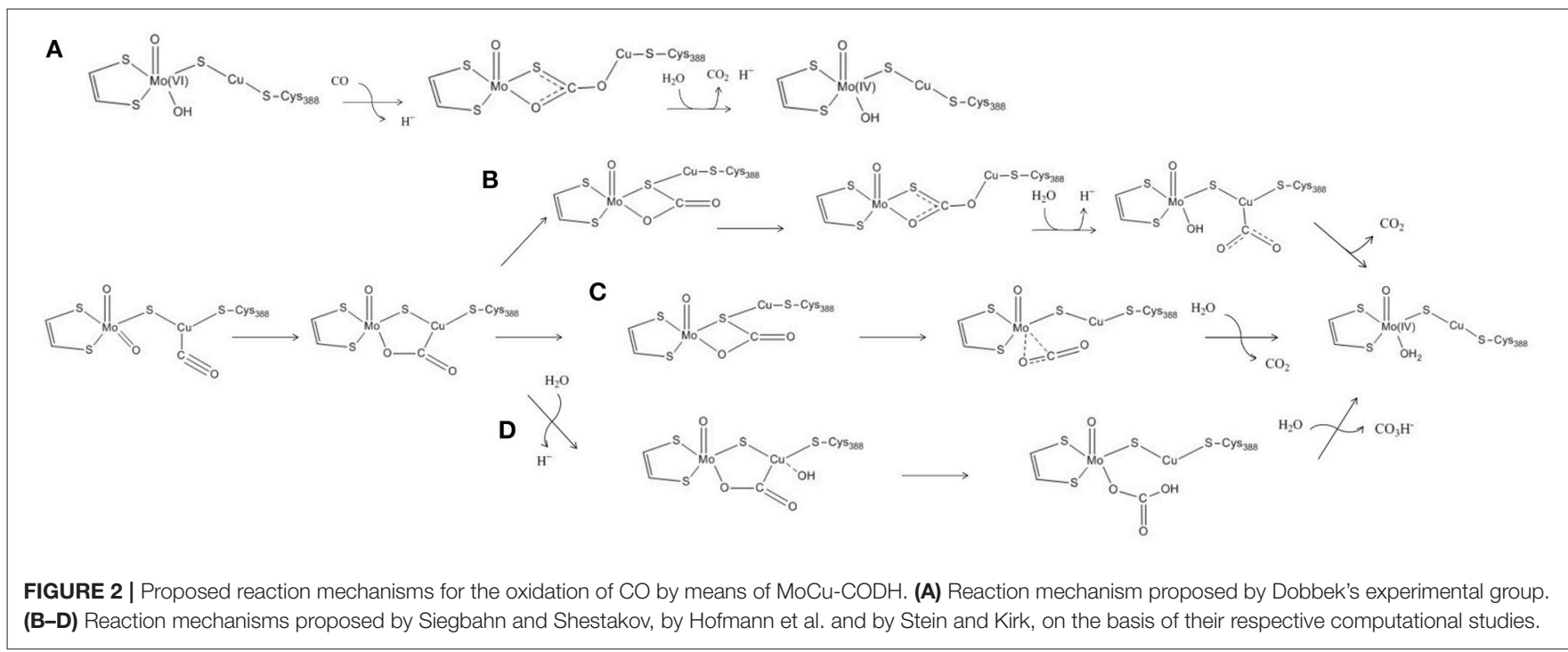


of about 20 atoms and a bigger one composed of about 70 atoms (see Figures $\mathbf{1 C , D}$ ). In the latter, some residues belonging to the second coordination spheres of the metals were explicitly included (Siegbahn and Shestakov, 2005). The hybrid density functional B3LYP (Lee et al., 1988; Becke, 1993) was employed in both cases to optimize the geometries and compute relative energies of intermediates along the putative catalytic cycles. For geometry optimizations, Hofmann and coworkers adopted the Lanl2DZ (Dunning and Hay, 1976; Hay and Wadt, 1985a,b; Wadt and Hay, 1985) effective core basis set with additional d-type functions on $\mathrm{S}$ atoms. This was followed by single point energy calculations using the SDD (Dunning, 1970; Dunning and Hay, 1977; Dolg et al., 1987) basis set augmented by d-type polarization functions on all non-hydrogen, non-metal atoms. The basis sets employed by Siegbahn and Shestakov were lacvp and lacv $3 p^{*}$-with ECP for $\mathrm{Mo}, \mathrm{Cu}$, and $\mathrm{S}$ atoms-for geometry optimizations and energy calculations, respectively. In both studies, the protein matrix surrounding the active site was modeled by a continuum dielectric with $\epsilon=4$ (Eckert and Klamt, 2002; Cossi et al., 2003).

A comparative analysis of the results coming from two such early investigations evidences a significant variability of the results obtained as a function of the adopted level of theory, within models of the same size. In particular, the calculated energy differences showed significant dependency on the basis sets used, with deviations up to $21 \mathrm{~kJ} / \mathrm{mol}$ in the case of intermediates involved in formation of the new $\mathrm{C}-\mathrm{O}$ bond, a key step in the catalytic process. Interestingly, previous studies of oxygen-atom transfer (OAT) reactions involving Mo-complexes (Li et al., 2013) and other transition-metal-containing systems (Hu and Chen, 2015; Li et al., 2015) also evidenced the strong basis set effect on computed energy differences along the reactive paths.

Notwithstanding the shortcomings deriving from the choice of basis sets, both Siegbahn, Shestakov and their respective coworkers evidenced a surprisingly high stability for the thiocarbonate intermediate. The presence of such a deep minimum on the energy landscape pertaining to the reaction mechanism was interpreted differently by the two groups. Siegbahn and Shestakov in particular proposed that the thiocarbonate derivative represents an intermediate of the COoxidation mechanism. However, in the proposed mechanism the barrier for the release of the $\mathrm{CO}_{2}$ product was estimated to be rather high, as it would require the insertion of a water molecule which was reported not to be a facile step (see Figure 2B). Differently, Hofmann and coworkers raised the possibility that the thiocarbonate adduct lies outside the catalytic cycle, in a deep potential energy well that would effectively slow down enzymatic activity (see Figure 2C). These authors further proposed that the constrains imposed by the protein matrix could prevent formation of such a stable offpath adduct, a hypothesis that- however-was later discarded as a result of a theoretical study focused on this topic (Siegbahn, 2011).

In a more recent theoretical study, a different mechanism for the oxidation of $\mathrm{CO}$ by the $\mathrm{MoCu}-\mathrm{CODH}$ enzyme was proposed (see Figure 2D) (Stein and Kirk, 2014). Using a cluster model analogous to the one previously employed by Hofmann and coworkers (see Figure 1B), at the PBE/TZP (Perdew et al., 1996; Ernzerhof and Scuseria, 1999) level of theory and including continuum dielectric contributions $(\epsilon=$ hexane) (Klamt and Schüürmann, 1993), Stein and Kirk proposed that the stable thiocarbonate intermediate formation could be bypassed by evolving bicarbonate as a final product rather than $\mathrm{CO}_{2}$. Bicarbonate formation would proceed via nucleophilic attack of a copper-activated water molecule on the $\mathrm{C}$ atom of the metalbound $\mathrm{CO}_{2}$. However, such a picture is at odds with recent experimental studies, which appear to exclude the possibility of forming a bicarbonate complex during catalysis (Dingwall et al., 2016).

Breglia and coworkers published the most recent theoretical study of $\mathrm{MoCu}-\mathrm{CODH}$, in which only the first shell coordination spheres were included in a QM model (see Figure 1C) (Breglia et al., 2017). Such a study mainly regards the hydrogenase activity of the enzyme and includes a comparative analysis of the binding reactions of the physiologic substrate-i.e., $\mathrm{CO}$-and of dihydrogen to the $\mathrm{Cu}$ ion. Similarly to previous studies on $\mathrm{H}_{2}$ - and CO-binding enzyme models in which a pure functional was used in conjuction with triple-zeta bases (Greco et al., 2015; Rovaletti and Greco, 2018), geometry optimizations and energy calculations were carried out in vacuo at BP86/def2-TZVP level (Perdew, 1986; Becke, 1988; Weigend and Ahlrichs, 2005). As far as the energetics of $\mathrm{CO}$ binding is concerned, the computed $\Delta \mathrm{E}$ was as negative as $-64 \mathrm{~kJ} / \mathrm{mol}$. A comparison of the latter value with the results of corresponding calculations previously performed at different levels of theory for the same reaction by Siegbahn, Hofmann and their respective coworkers, evidences significant discrepancies ( $\Delta \Delta \mathrm{E}=50$ and $31 \mathrm{~kJ} / \mathrm{mol}$, respectively). Actually, the occurrence of such large differences comes with little surprise, given the well-known shortcomings in binding energy calculations using quantum chemical models of coordination complexes and their ligands (Husch et al., 2018).

\section{LARGE QM-CLUSTER MODELS AND HYBRID MODELS}

The importance of extending the dimension of the bimetallic active site model and systematically accounting for the effects of the second-sphere residues on energetics was evidenced in a recent theoretical work by Rokhsana and coworkers (Rokhsana et al., 2016). In fact, a large-size cluster model of around 180 atoms (see Figure 1E) turned out to be required for a fully satisfactory reproduction of experimentally determined structural parameters. The same held true for the evaluation of the most plausible protonation states of the $\mathrm{Mo} / \mathrm{Cu}$ core, which was done taking into account key geometric features of the enzyme crystal structure and redox potential measurements available in literature. The authors were able to assess in particular the protonation state of the equatorial oxo ligand of $\mathrm{Mo}$, at the different redox states attained during catalysis. As for the adopted level of theory, Rokhsana et al. employed the def2-TZVP basis set for all elements, apart from $\mathrm{H}$ and $\mathrm{C}$ atoms, for which the def2-SVP basis set was used (Weigend and Ahlrichs, 2005). The protein environment was modeled by a continuum dielectric with $\epsilon=4$ 
(Klamt and Schüürmann, 1993). The BP86 and B3LYP density functionals were used for geometry optimizations and energy evaluation, respectively.

In a subsequent study, explicit consideration of the whole protein environment was achieved by means of a hybrid quantum mechanics/molecular mechanics (QM/MM) approach (Xu and Hirao, 2018). In the work of $\mathrm{Xu}$ and Hirao, the active site was described by a QM region of 89 atoms (see Figure 1F) using the B3LYP functional. During geometry optimization, the SDD effective core potential basis set was employed to represent the transition metal ions, whereas the $6-31 \mathrm{G}^{*}$ basis set was adopted for all the other atoms (Dolg et al., 1987; Andrae et al., 1990). Single-point energy calculations were carried out using the larger def2-TZVP basis set. For the molecular mechanics calculations, the AMBER03 force field was employed (Duan et al., 2003). Moreover, the Grimme's D3-correction with Becke-Johnson damping $[\mathrm{D} 3(\mathrm{BJ})]$ was taken into account in the calculations (Grimme et al., 2011). The previously proposed catalytic mechanisms that involve the formation of the thiocarbonate species were re-investigated at such a level of theory. According to Xu and Hirao's study, the S-C-bound adduct would be formed along the reaction pathway as previously suggested (Dobbek et al., 2002; Siegbahn and Shestakov, 2005). However, based on the novel QM/MM results, the thiocarbonate species thus formed would not be as stable as previously proposed. It has to be remarked that the thiocarbonate intermediate was not found to be directly linked to the transition state for $\mathrm{CO}_{2}$ releasing. In fact, it was proposed that - after thiocarbonate formationthe reaction needs to follow a reverse process to productively proceed toward $\mathrm{CO}_{2}$ evolution. Notably, the overall barrier for the proposed catalytic mechanism was found to be low (in the order of $50 \mathrm{~kJ} / \mathrm{mol}$ ). In the same study, Xu and Hirao also carried out purely QM calculations with a QM-cluster of the same size of the quantum-mechanical region of their hybrid model, and compared the obtained results with those coming from QM/MM modeling. Such a comparison evidenced that the protein environment is not involved in modulating the kinetic barrier associated with the investigated catalytic mechanism. However, it was found that the protein matrix plays an important role in the stabilization of the $\mathrm{CO}_{2}$-released state. Finally, it was also reported that the inclusion of dispersive corrections lowers by $15 \mathrm{~kJ} / \mathrm{mol}$ the activation barrier of the product-releasing step, in line with what was expected for the modeling of a bimolecular reaction step.

\section{CONCLUDING REMARKS}

Over the last fifteen years, the theoretical investigation of the $\mathrm{CO}$ oxidation mechanism by $\mathrm{MoCu}-\mathrm{CODH}$ has given rise to a debate, the essentials of which are centered on the possible occurrence and on the role of a thiocarbonate catalytic intermediate. In the above sections, we have reported key details of the various computational studies published to date, and we are now in the condition to present a more general outlook on the state of the art regarding $\mathrm{MoCu}-\mathrm{CODH}$.

The early studies by Siegbahn, Hofmann, and their respective coworkers evidenced that the thiocarbonate intermediate would occupy a deep well in the energy profiles pertaining to the investigated reaction mechanisms. However, the kinetic barriers they computed for $\mathrm{CO}_{2}$ evolution were at least $30 \mathrm{~kJ} / \mathrm{mol}$ higher than the recently determined experimental counterpart (Zhang et al., 2010). In part, this picture depends on the neglect of dispersive corrections: their inclusion became a standard possibility only after the publication of the mentioned study (Siegbahn, 2011).

Results more compatible with the experimental evidence of a kinetic barrier of around $50 \mathrm{~kJ} / \mathrm{mol}$ were obtained by $\mathrm{Xu}$ and Hirao, who exploited a larger QM-cluster model with the explicit inclusion of most of the second-sphere coordination environment, along with employment of large basis sets and dispersion corrections (Xu and Hirao, 2018). It is noticeable that, according to $\mathrm{Xu}$ and Hirao's results, the thiocarbonate species still appears to behave as a thermodynamic sink. Even though not a very deep one, such a sink would effectively hamper the advancement along the proposed path toward products, a rather unusual role for a species formed during an enzymatic process.

All the catalytic mechanisms proposed in the theoretical studies reviewed here focus on the possibility that the Mobound equatorial oxo ligand performs the nucleophilic attack on the activated $\mathrm{CO}$ substrate bound to the $\mathrm{Cu}$ ion. This is in line with what has been suggested in the case of the catalytic mechanism of the homologous xanthine oxidases enzymes. However, a variant with respect to such a picture has been recently proposed, in which an activated water molecule would play the role of nucleophile (Hille et al., 2015). Notably, to the best of our knowledge this alternative mechanism has not yet been investigated by QM or hybrid QM/MM studies.

Future theoretical studies on this (and other) putative catalytic mechanism will possibly face the challenge associated with rather pronounced fluctuations of computed energy differences as a function of the adopted level of theory. As far as the application of density functional theory is concerned, extensive benchmarking could in principle help to improve the theoretical predictions. In this regard, the available experimental data on enzyme inhibition - by thiols in particular-could represent a useful dataset, keeping in mind that the reproduction of binding energies in the case of bulky thiols might require extensive protein matrix phase space sampling in the case of QM/MM studies. High level $a b$ initio methods are a viable-though still challenging-alternative for providing reliable results. In fact, thanks to recent methodological developments, the treatment of relatively large bimetallic systems has been shown to be computationally affordable with multiconfigurational postHartree-Fock approaches (Phung et al., 2016, 2018; Dong et al., 2017).

The relevance of $\mathrm{MoCu}-\mathrm{CODH}$ as an inspiring system for future biomimetic and bioengineering applications is currently growing. This is due not only to the relevance of the reactions it catalyzes, but also to its resistance to atmospheric $\mathrm{O}_{2}$ exposurea rare feature in the case of enzymes expressing carbon monoxide dehydrogenase and hydrogenase activities (Choi et al., 2017; Gourlay et al., 2018; Groysman et al., 2018). Notably, the recent establishment of a functional heterologous expression system for 
the MoCu-CODH enzyme (Kaufmann et al., 2018) together with developments in the computational chemistry field will hopefully boost the positive feedback among biochemical, biomimetic and quantum chemical studies, opening new perspectives for a deeper understanding of this interesting metalloenzyme.

\section{REFERENCES}

Andrae, D., Haeussermann, U., Dolg, M., Stoll, H., and Preuss, H. (1990). Energyadjusted $a b$ initio pseudopotentials for the second and third row transition elements. Theor. Chim. Acta 77, 123-141.

Becke, A. D. (1988). Density-functional exchange-energy approximation with correct asymptotic behavior. Phys. Rev. A 38:3098.

Becke, A. D. (1993). A new mixing of Hartree-Fock and local density-functional theories. J. Chem. Phys. 98, 1372-1377.

Breglia, R., Bruschi, M., Cosentino, U., De Gioia, L., Greco, C., Miyake, T., et al. (2017). A theoretical study on the reactivity of the $\mathrm{Mo} / \mathrm{Cu}$-containing carbon monoxide dehydrogenase with dihydrogen. Protein Eng. Design Select. 30, 169-174. doi: 10.1093/protein/gzw071

Choi, E. S., Min, K., Kim, G.-J., Kwon, I., and Kim, Y. H. (2017). Expression and characterization of Pantoea CO dehydrogenase to utilize CO-containing industrial waste gas for expanding the versatility of $\mathrm{CO}$ dehydrogenase. Sci. Rep. 7:44323. doi: $10.1038 /$ srep44323

Cossi, M., Rega, N., Scalmani, G., and Barone, V. (2003). Energies, structures, and electronic properties of molecules in solution with the C-PCM solvation model. J. Comput. Chem. 24, 669-681. doi: 10.1002/jcc.10189

Dingwall, S., Wilcoxen, J., Niks, D., and Hille, R. (2016). Studies of carbon monoxide dehydrogenase from Oligotropha carboxidovorans. J. Mol. Catal. B Enzymat. 134, 317-322. doi: 10.1016/j.molcatb.2016.10.007

Dobbek, H., Gremer, L., Kiefersauer, R., Huber, R., and Meyer, O. (2002). Catalysis at a dinuclear $[\mathrm{CuSMo}(=\mathrm{O}) \mathrm{OH}]$ cluster in a $\mathrm{CO}$ dehydrogenase resolved at 1.1-Å resolution. Proc. Natl. Acad. Sci. U.S.A. 99, 15971-15976. doi: $10.1073 /$ pnas. 212640899

Dolg, M., Wedig, U., Stoll, H., and Preuss, H. (1987). Energy-adjusted ab initio pseudopotentials for the first row transition elements. J. Chem. Phys. 86, 866-872.

Dong, G., Phung, Q. M., Hallaert, S. D., Pierloot, K., and Ryde, U. (2017). $\mathrm{H}_{2}$ binding to the active site of [NiFe] hydrogenase studied by multiconfigurational and coupled-cluster methods. Phys. Chem. Chem. Phys. 19, 10590-10601. doi: 10.1039/C7CP01331K

Duan, Y., Wu, C., Chowdhury, S., Lee, M. C., Xiong, G., Zhang, W., et al. (2003). A point-charge force field for molecular mechanics simulations of proteins based on condensed-phase quantum mechanical calculations. J. Comput. Chem. 24, 1999-2012. doi: 10.1002/jcc.10349

Dunning, T. and Hay, P. (1976). Modern Theoretical Chemistry. Plenum, NY: HF Schaefer III.

Dunning, T. H., and Hay, P. J. (1977). "Gaussian basis sets for molecular calculations," in Methods of Electronic Structure Theory, ed H. F. Schaefer (Boston, MA: Springer), 1-27.

Dunning, T. H. Jr. (1970). Gaussian basis functions for use in molecular calculations. I. contraction of (9s5p) atomic basis sets for the first-row atoms. J. Chem. Phys. 53, 2823-2833.

Eckert, F., and Klamt, A. (2002). Fast solvent screening via quantum chemistry: COSMO-RS approach. AIChE J. 48, 369-385. doi: 10.1002/aic.6904 80220

Ernzerhof, M., and Scuseria, G. E. (1999). Assessment of the PerdewBurke-Ernzerhof exchange-correlation functional. J. Chem. Phys. 110, 5029-5036.

Gnida, M., Ferner, R., Gremer, L., Meyer, O., and Meyer-Klaucke, W. (2003). A novel binuclear [CuSMo] cluster at the active site of carbon monoxide dehydrogenase: characterization by $\mathrm{X}$-ray absorption spectroscopy. Biochemistry 42, 222-230. doi: 10.1021/bi026514n

Gourlay, C., Nielsen, D. J., Evans, D. J., White, J. M., and Young, C. G. (2018). Models for aerobic carbon monoxide dehydrogenase: synthesis,

\section{AUTHOR CONTRIBUTIONS}

All authors listed have made a substantial, direct and intellectual contribution to the work, and approved it for publication.

characterization and reactivity of paramagnetic $\mathrm{Mo}^{\mathrm{V}} \mathrm{O}(\mu-\mathrm{S}) \mathrm{Cu}^{\mathrm{I}}$ complexes. Chem. Sci. 9, 876-888. doi: 10.1039/c7sc04239f

Gourlay, C., Nielsen, D. J., White, J. M., Knottenbelt, S. Z., Kirk, M. L., and Young, C. G. (2006). Paramagnetic active site models for the molybdenum-copper carbon monoxide dehydrogenase. J. Am. Chem. Soc. 128, 2164-2165. doi: 10.1021/ja056500f

Greco, C., Ciancetta, A., Bruschi, M., Kulesza, A., Moro, G., and Cosentino, U. (2015). Influence of key amino acid mutation on the active site structure and on folding in acetyl-CoA synthase: a theoretical perspective. Chem. Commun. 51, 8551-8554. doi: 10.1039/c5cc01575h

Grimme, S., Ehrlich, S., and Goerigk, L. (2011). Effect of the damping function in dispersion corrected density functional theory. J. Comput. Chem. 32, 14561465. doi: $10.1002 /$ jcc. 21759

Groysman, S., Hollingsworth, T., Hollingsworth, R. L., and Lord, R. L. (2018). Cooperative bimetallic reactivity of a heterodinuclear molybdenumcopper model of Mo-Cu CODH. Dalton Trans. 47, 10017-10024. doi: $10.1039 / \mathrm{c} 8 \mathrm{dt} 02323 \mathrm{a}$

Hay, P. J., and Wadt, W. R. (1985a). Ab initio effective core potentials for molecular calculations. Potentials for $\mathrm{K}$ to $\mathrm{Au}$ including the outermost core orbitals. $J$. Chem. Phys. 82, 299-310. doi: 10.1063/1.448975

Hay, P. J., and Wadt, W. R. (1985b). Ab initio effective core potentials for molecular calculations. Potentials for the transition metal atoms Sc to Hg. J. Chem. Phys. 82, 270-283. doi: $10.1063 / 1.448799$

Hille, R. (2013). The molybdenum oxotransferases and related enzymes. Dalton Trans. 42, 3029-3042. doi: 10.1039/c2dt32376a

Hille, R., Dingwall, S., and Wilcoxen, J. (2015). The aerobic CO dehydrogenase from Oligotropha carboxidovorans. J. Biol. Inorgan. Chem. 20, 243-251. doi: 10.1007/s00775-014-1188-4

Hofmann, M., Kassube, J. K., and Graf, T. (2005). The mechanism of $\mathrm{Mo} / \mathrm{Cu}$ dependent CO dehydrogenase. J. Biol. Inorgan. Chem. 10, 490-495. doi: $10.1007 / \mathrm{s} 00775-005-0661-5$

Hu, L., and Chen, H. (2015). Assessment of DFT methods for computing activation energies of Mo/W-mediated reactions. J. Chem. Theor. Comput. 11, 4601-4614. doi: $10.1021 /$ acs.jctc. 5 b00373

Husch, T., Freitag, L., and Reiher, M. (2018). Calculation of ligand dissociation energies in large transition-metal complexes. J. Chem. Theor. Comput. 14, 2456-2468. doi: 10.1021/acs.jctc. 8 b00061

Kaufmann, P., Duffus, B. R., Teutloff, C., and Leimkuhler, S. (2018). Functional studies on oligotropha carboxidovorans molybdenum-copper CO dehydrogenase produced in Escherichia coli. Biochemistry 57, 2889-2901. doi: 10.1021 /acs.biochem. 8 b00128

Klamt, A., and Schüürmann, G. (1993). COSMO: a new approach to dielectric screening in solvents with explicit expressions for the screening energy and its gradient. J. Chem. Soc. Perkin Trans. 2, 799-805. doi: 10.1039/P29930000799

Kreß, O., Gnida, M., Pelzmann, A. M., Marx, C., Meyer-Klaucke, W., and Meyer, O. (2014). Reversible inactivation of CO dehydrogenase with thiol compounds. Biochem. Biophys. Res. Commun. 447, 413-418. doi: 10.1016/j.bbrc.2014.03.147

Lee, C., Yang, W., and Parr, R. G. (1988). Development of the Colle-Salvetti correlation-energy formula into a functional of the electron density. Phys. Rev. B 37:785. doi: 10.1103/PhysRevB.37.785

Li, J., Andrejić, M., Mata, R. A., and Ryde, U. (2015). A computational comparison of oxygen atom transfer catalyzed by dimethyl sulfoxide reductase with Mo and W. Eur. J. Inorgan. Chem. 2015, 3580-3589. doi: 10.1002/ejic.201500209

Li, J.-L., Mata, R. A., and Ryde, U. (2013). Large density-functional and basis-set effects for the DMSO reductase catalyzed oxo-transfer reaction. J. Chem. Theor. Comput. 9, 1799-1807. doi: 10.1021/ct301094r

Liu, L., Zhuang, Q., Zhu, Q., Liu, S., van Asperen, H., and Pihlatie, M. (2018). Global soil consumption of atmospheric carbon monoxide: an analysis 
using a process-based biogeochemistry model. Atmos. Chem. Phys. 18:7913. doi: 10.5194/acp-18-7913-2018

Perdew, J. P. (1986). Density-functional approximation for the correlation energy of the inhomogeneous electron gas. Phys. Rev. B 33:8822. doi: 10.1103/PhysRevB.33.8822

Perdew, J. P., Burke, K., and Ernzerhof, M. (1996). Generalized gradient approximation made simple. Phys. Rev. Lett. 77:3865. doi: 10.1103/PhysRevLett.77.3865

Phung, Q. M., Domingo, A., and Pierloot, K. (2018). Dinuclear iron(II) spincrossover Compounds: a theoretical study. Chem. A Eur. J. 24, 5183-5190. doi: 10.1002/chem.201704441

Phung, Q. M., Wouters, S., and Pierloot, K. (2016). Cumulant approximated second-Order perturbation theory based on the density matrix renormalization group for transition metal complexes: a benchmark study. J. Chem. Theor. Comput. 12, 4352-4361. doi: 10.1021/acs.jctc.6b00714

Rokhsana, D., Large, T. A., Dienst, M. C., Retegan, M., and Neese, F. (2016). A realistic in silico model for structure/function studies of molybdenum-copper CO dehydrogenase. J. Biol. Inorgan. Chem. 21, 491-499. doi: 10.1007/s00775-016-1359-6

Rovaletti, A., and Greco, C. (2018). Organophosphorous ligands in hydrogenaseinspired iron-based catalysts: a DFT study on the energetics of metal protonation as a function of P-atom substitution. J. Phys. Org. Chem. 31:e3748. doi: $10.1002 /$ poc. 3748

Santiago, B., and Meyer, O. (1996). Characterization of hydrogenase activities associated with the molybdenum CO dehydrogenase from Oligotropha carboxidovorans. FEMS Microbiol. Lett. 136, 157-162. doi: 10.1111/j.1574-6968.1996.tb08042.x

Shanmugam, M., Wilcoxen, J., Habel-Rodriguez, D., Cutsail III, G. E., Kirk, M. L., Hoffman, B. M., et al. (2013). 13C and 63,65Cu ENDOR studies of CO Dehydrogenase from Oligotropha carboxidovorans. Experimental evidence in support of a copper-carbonyl intermediate. J. Am. Chem. Soc. 135, 1777517782. doi: $10.1021 /$ ja406136f

Siegbahn, P. E. (2011). The effect of backbone constraints: the case of water oxidation by the oxygen-evolving complex in PSII. ChemPhysChem 12, 32743280. doi: $10.1002 /$ cphc. 201100475
Siegbahn, P. E. M., and Shestakov, A. F. (2005). Quantum chemical modeling of $\mathrm{CO}$ oxidation by the active site of molybdenum $\mathrm{CO}$ dehydrogenase. J. Comput. Chem. 26, 888-898. doi: 10.1002/jcc.20230

Stein, B. W., and Kirk, M. L. (2014). Orbital contributions to CO oxidation in Mo-Cu carbon monoxide dehydrogenase. Chem. Commun. 50, 1104-1106. doi: 10.1039/C3CC47705C

Wadt, W. R., and Hay, P. J. (1985). Ab initio effective core potentials for molecular calculations. Potentials for main group elements Na to Bi. J. Chem. Phys. 82, 284-298. doi: 10.1063/1.448800

Weigend, F., and Ahlrichs, R. (2005). Balanced basis sets of split valence, triple zeta valence and quadruple zeta valence quality for h to rn: design and assessment of accuracy. Phys. Chem. Chem. Phys. 7, 3297-3305. doi: 10.1039/b508541a

Wilcoxen, J., and Hille, R. (2013). The hydrogenase activity of the molybdenum/copper-containing carbon monoxide dehydrogenase of oligotropha carboxidovorans. J. Biol. Chem. 288, 36052-36060. doi: $10.1074 /$ jbc.M113.522441

$\mathrm{Xu}, \mathrm{K}$., and Hirao, H. (2018). Revisiting the catalytic mechanism of Mo-Cu carbon monoxide dehydrogenase using QM/MM and DFT calculations. Phys. Chem. Chem. Phys. 20, 18938-18948. doi: 10.1039/C8CP00858B

Zhang, B., Hemann, C. F., and Hille, R. (2010). Kinetic and spectroscopic studies of the molybdenum-copper CO dehydrogenase from Oligotropha carboxidovorans. J. Biol. Chem. 285, 12571-12578. doi: $10.1074 /$ jbc.M109.076851

Conflict of Interest Statement: The authors declare that the research was conducted in the absence of any commercial or financial relationships that could be construed as a potential conflict of interest.

Copyright (c) 2019 Rovaletti, Bruschi, Moro, Cosentino and Greco. This is an openaccess article distributed under the terms of the Creative Commons Attribution License (CC BY). The use, distribution or reproduction in other forums is permitted, provided the original author(s) and the copyright owner(s) are credited and that the original publication in this journal is cited, in accordance with accepted academic practice. No use, distribution or reproduction is permitted which does not comply with these terms. 